OPEN ACCESS

Edited by:

Thomas Deller,

Goethe-University, Germany

Reviewed by:

Imre Vida,

Charité Universitätsmedizin Berlin,

Germany

Eckart D. Gundelfinger, Leibniz Institute for Neurobiology,

Germany

*Correspondence: Walter Volknandt volknandt@bio.uni-frankfurt.de

Received: 24 November 2016 Accepted: 07 February 2017 Published: 20 February 2017

Citation:

Weingarten J, Weingarten $\mathrm{M}$, Wegner $M$ and Volknandt $W$ (2017) APP-A Novel Player within the Presynaptic Active Zone Proteome.

Front. Mol. Neurosci. 10:43. doi: 10.3389/fnmol.2017.00043

\section{APP-A Novel Player within the Presynaptic Active Zone Proteome}

\author{
Jens Weingarten ${ }^{1}$, Melanie Weingarten ${ }^{1}$, Martin Wegner ${ }^{2}$ and Walter Volknandt ${ }^{1 \text { * }}$ \\ ${ }^{1}$ Institute for Cell Biology and Neuroscience, Biologicum and BMLS, Goethe University, Frankfurt am Main, Germany, \\ ${ }^{2}$ Department of Molecular Bioinformatics, Goethe University, Frankfurt am Main, Germany
}

The amyloid precursor protein (APP) was discovered in the 1980s as the precursor protein of the amyloid A4 peptide. The amyloid A4 peptide, also known as A-beta $(A \beta)$, is the main constituent of senile plaques implicated in Alzheimer's disease (AD). In association with the amyloid deposits, increasing impairments in learning and memory as well as the degeneration of neurons especially in the hippocampus formation are hallmarks of the pathogenesis of $A D$. Within the last decades much effort has been expended into understanding the pathogenesis of $A D$. However, little is known about the physiological role of APP within the central nervous system (CNS). Allocating APP to the proteome of the highly dynamic presynaptic active zone (PAZ) identified APP as a novel player within this neuronal communication and signaling network. The analysis of the hippocampal PAZ proteome derived from APP-mutant mice demonstrates that APP is tightly embedded in the underlying protein network. Strikingly, APP deletion accounts for major dysregulation within the PAZ proteome network. $\mathrm{Ca}^{2+}$-homeostasis, neurotransmitter release and mitochondrial function are affected and resemble the outcome during the pathogenesis of $\mathrm{AD}$. The observed changes in protein abundance that occur in the absence of APP as well as in AD suggest that APP is a structural and functional regulator within the hippocampal PAZ proteome. Within this review article, we intend to introduce APP as an important player within the hippocampal PAZ proteome and to outline the impact of APP deletion on individual PAZ proteome subcommunities.

Keywords: amyloid precursor protein, hippocampus, neuronal network, presynaptic active zone, synapse

\section{INTRODUCTION}

The development of a neuronal circuit requires precise coordination of billions of neurons, with up to 100,000 synaptic connections each, forming a stable but plastic network and persisting over the lifespan of an organism (Turrigiano, 2008, 2012). The key word deciphering this phenomenon is "homeostasis" and was introduced by Walter Cannon in the early 1930's (Cannon, 1932). Within the neuronal network numerous homeostatic mechanisms ensure physiological activity in a spatio-temporal manner on various groups of synapses (Turrigiano, 2008; Yu and Goda, 2009). Maintenance of synaptic homeostasis demands on a coordinated proteomic response at both-pre- and postsynaptic sites (Schanzenbächer et al., 2016).

\footnotetext{
Abbreviations: AD, Alzheimer's disease; APLP1/2, amyloid precursor like proteins1 and 2; APP, amyloid precursor protein; CA, cornu ammonis; HD, Huntington's disease; PAZ, presynaptic active zone; PD, Parkinson's disease; SNAP25, synaptosomal associated protein 25; SV2, synaptic vesicle protein 2; VAMP2, vesicle associated membrane protein2/synaptobrevin2.
} 
Consecutive steps of processing the arrival of an action potential into a chemical signal by recruiting a subset of individual proteins that fuse synaptic vesicles with the presynaptic plasma membrane, release of their neurotransmitter into the synaptic cleft, which further react with their specific receptor at the postsynapse (Figure 1), demands on a rather stringent progression. Key players within this network comprise prominent candidates like synaptic vesicle protein 2 (SV2), synaptotagmin-1, synaptosomal associated protein 25 (SNAP25), syntaxin and vesicle associated membrane protein2/synaptobrevin2 (VAMP2; Südhof and Rizo, 2011; Südhof, 2012; Laßek et al., 2015). The unique set of proteins regulating, mediating and controlling proper presynaptic physiology was recently complemented by a yet unappreciated companion - the amyloid precursor protein (APP).

\section{APP-A BRIEF PROFILE}

The APP belongs to an evolutionary conserved gene family with specific expression pattern in C. elegans, Drosophila and mammals (reviewed in Coulson et al., 2000; Jacobsen and Iverfeldt, 2009). Discovered during the 1980s as precursor protein of A-beta $(A \beta)$-the main constituent of senile plaques-much effort has been made to understand the pathophysiology of Alzheimer's disease (AD) and the physiological function of APP (Glenner and Wong, 1984; Kang et al., 1987). The progression of $\mathrm{AD}$ is characterized by a massive loss of synapses especially within the hippocampus. Extracellular senile plaques and intracellular neurofibrillary tangles induce and promote successive degeneration of neurons manifested by severe impairments in learning and memory and behavioral changes (Grundke-Iqbal et al., 1986; Supnet and Bezprozvanny, 2010). Enzymatic processing of APP is initiated by either $\beta$-secretase $/ \gamma$-secretase (amyloidogenic pathway) cleavage, or $\alpha$-secretase $/ \gamma$ - secretase (non-amyloidogenic pathway). The proteolytic processing of amyloid precursor like proteins 1 and 2 (APLP1 and APLP2) is comparable to that of APP, however, only the amyloidogenic pathway can induce the formation of $\mathrm{A} \beta$-peptides (Eggert et al., 2004). Until now, little is known about the shift in enzymatic processing of the APP leading to
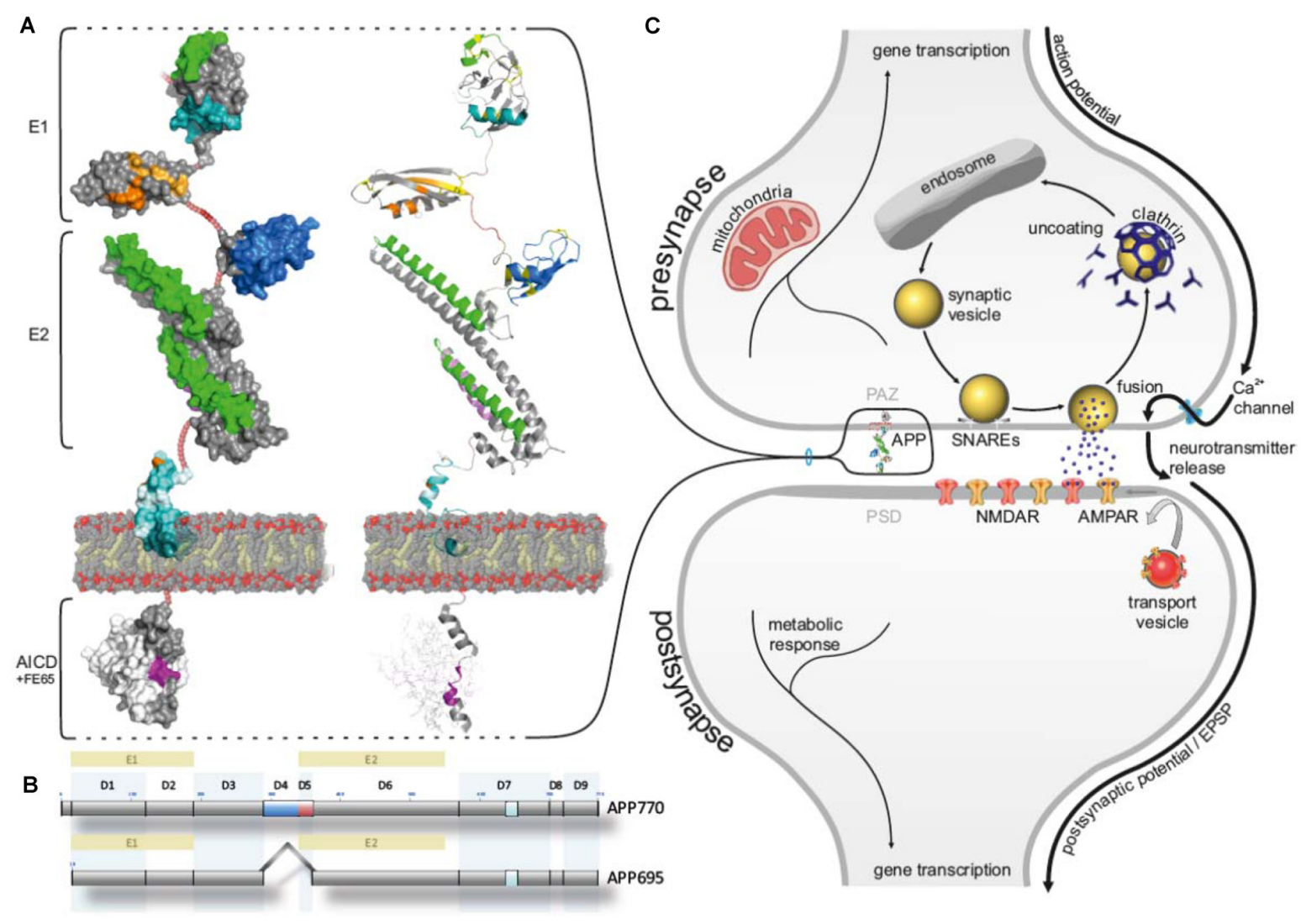

FIGURE 1 | Amyloid precursor protein (APP) allocated to the presynaptic active zone (PAZ). (A) Schematic surface illustration (left) and cartoon (right) representing the domain organization of APP (modeling structures are created by PyMOL Molecular Graphics system based on X-ray data from protein data base, PDB; 11.2010). Heparin-binding domain/ growth factor-like domain (HBD/GFLD, green), disulfide bridges (yellow), copper-binding domain (CuBD, orange), zinc-binding domain ( $\mathrm{nBBD}$, bright orange), acidic domain ( $\mathrm{DE}$, firebrick), Kunitz protease inhibitor domain (blue), collagene binding domain (violet), A-beta (A $\beta$ ) sequence (cyan), secretase cleavage site (pale cyan), transmembrane segment (dark teal), YENPTY sequence (magenta), NPXY sequence (purple), FE65 adaptor protein (white), non-modeled render sequences (ruby). (B) Subdivision of domains (D1-D9) in APP695 (expressed in neurons) and APP770 (KPI highlighted in blue, acidic domain highlighted in blue). (C) Schematic cartoon of a chemical synapse highlighting APP at the PAZ. 
the accumulation of $A \beta$-peptides and the formation of oligomers and fibrils. Since senile plaques consist mainly of $A \beta$-fibrils, it was of great interest how these structures are organized (Lu et al., 2013). The analysis of these fibrils derived from AD patients revealed an individual molecular structure. These variations were suggested to correlate with the severity of impairments in the individual pathogenesis of $\mathrm{AD}$ in patients ( $\mathrm{Lu}$ et al., 2013).

APP is a type 1 transmembrane protein with a large $\mathrm{N}$-terminal domain, a single transmembrane region and a short C-terminal domain (Figure 1). The N-terminal domain is subdivided into an E1 domain comprising a heparinbinding/growth factor-like domain (HBD/GFLD), a copper and zinc-binding domain ( $\mathrm{CuBD} / \mathrm{ZnBD})$ followed by an acidic region (DE), optionally a KPI-domain (not present in the neuronal specific isoform APP695), and an E2 domain consisting of a second $\mathrm{HBD}$ (HBD2) a collagen-binding region and $\mathrm{N}$-glycosylation binding sites (Jacobsen and Iverfeldt, 2009; Kaden et al., 2012). The APP intracellular domain (AICD) contains the highly conserved YENPTY motif involved in the internalization of APP and phosphorylated or dephosphorylated tyrosine mediated binding of adaptor proteins like FE65 (Figure 1), Dab1 and X11a (munc-18 interacting protein, Mint; Jacobsen and Iverfeldt, 2009). All APP family members reveal a high structural overlap except the $\mathrm{A} \beta$ domain that is only present in mammalian APP.

\section{APP AT THE SYNAPSE}

Multiple isoforms were described for mammalian APP (e.g., 695aa, 770aa), but only APP695aa is expressed in neurons. Within neurons, APP was discussed as bona fide SV (Groemer et al., 2011) and constituent of the presynaptic plasma membrane (Marquez-Sterling et al., 1997; Lyckman et al., 1998). In addition, APP was described as a constituent of endocytosed synaptic vesicles, but being sorted away from bona fide synaptic vesicles (Marquez-Sterling et al., 1997). On the contrary, Groemer et al. (2011) reported a small copy number of APP to synaptic vesicles as a result of endosomal synaptic vesicle recycling processes. However, they emphasized that the majority of APP was immunodetected in fractions containing the plasma membrane, and only a small amount was present in purified synaptic vesicle fractions (Groemer et al., 2011). In our studies, we clearly demonstrated that APP and its family members are constituents of the presynaptic plasma membrane and that APP is virtually absent from synaptic vesicles (Laßek et al., 2013). Within the presynaptic nerve terminal, a small section characterized by an assembly of electron dense material, is responsible for $\mathrm{Ca}^{2+}$-triggered exocytosis of synaptic vesicles. This section is called presynaptic active zone (PAZ; Gray, 1963; Südhof, 2012). The composition of the PAZ proteome identified the release site as dynamic focal hot spot, providing the prerequisite for structural and functional changes also in the adult nerve terminal. Neuronal communication and signal transduction depends not only on the concerted action of individual proteins within the PAZ but also on proper energy supply (Boveris and Navarro,
2008). Besides the glycolytic chain associated with synaptic vesicles (reviewed in Burré and Volknandt, 2007), mitochondria are the main source for the production of ATP at the presynaptic terminal. Therefore, mitochondria are essential in maintaining presynaptic homeostasis and phosphorylation reactions and are highly involved in synaptic plasticity (reviewed in Mattson et al., 2008). The allocation of APP to the proteome of this highly dynamic substructure of the presynapse, identified APP as yet unknown player within the neuronal communication and signaling network (Laßek et al., 2013, 2016).

To address the question which physiological function APP is executing in the central nervous system (CNS), a variety of genetically designed mouse models has been generated (Heber et al., 2000; Ring et al., 2007; Hick et al., 2015). It turned out that loss of APP causes an age-dependent phenotype with no severe physiological impairments in younger mice but impairments in learning and memory in the elderly (Phinney et al., 1999; Ring et al., 2007). At postsynaptic sites, reduced dendritic length and branching accompanied by a total spine density reduction was characteristic for old APP-KO mice and indicates a physiological role of APP in maintaining spine density (Tyan et al., 2012). This was further supported by Weyer et al. (2014) demonstrating a specific role of APP in sustaining spine structure and density. Classification of spine structure can be morphologically addressed revealing stubby, thin and mushroom spines. In APP-KO cornu ammonis 1 (CA1) neurons this spine subtype distribution is altered by a significant decrease in mushroom spines (Weyer et al., 2014). Interestingly, substantial changes of the proteomic composition of neurotransmitter release sites are already detectable in younger mice (Laßek et al., 2014, 2016). Since APP plays an essential role during the development of the neuronal circuit (Lazarov and Demars, 2012), it was suggested that the APLP2 compensates for the loss of APP (Weyer et al., 2011; Laßek et al., 2016). Screening immunopurified PAZs derived from individual total mouse brain revealed prominent players to be affected by APP deletion. Candidates like SV2A, synaptotagmin-1 and synaptophysin turned out to be differentially regulated. It is worth mentioning, that the opposite effect was observed for deletion of either APLP1 or APLP2 (Laßek et al., 2014). Moreover, deletion of the family members did not result in any morphological alterations in CNS or overall impairments in learning and memory (Heber et al., 2000; Weyer et al., 2011). Memory formation requires a variety of network oscillations that are regularly synchronized between hippocampal CA1 and CA3 region (Korte et al., 2012). As inhibitory interneurons play an essential role in this coordinated action of synchronization, their oscillations can affect a large population of pyramidal neurons, inhibiting specific input pathways and guarantee for a high background-to-noise ratio (Mann and Paulsen, 2007). Accompanied with the observed shift in the excitatory-inhibitory ratio in APP-KO mice, it was suggested that GABAA receptormediated inhibition is altered in aged APP-KO mice and that these changes contribute to the reduction in LTP in aged APP-KO mice. This assumption was further sustained by 
LTP-rescue experiments employing pharmacological blockade of $\mathrm{GABA}_{\mathrm{A}}$ receptors (Fitzjohn et al., 2000; Korte et al., 2012). Synaptic plasticity requires persistent changes within the entire network. Thereby, the strength of a neuronal connection is individually adjusted (up or down) dependent on homeostatic synaptic scaling (Turrigiano, 2012; Davis, 2013). Homeostasis implies the capability to restore individual baseline functions upon continued input. This is achieved by controlling and modulating the expression and trafficking of specific proteins and protein complexes. Initially, synaptic scaling was described as bidirectional modulation of neurotransmitter receptor abundance at individual synapses. In this context, it was suggested that this effect stabilizes neuronal excitability while sustaining learning-related information (Turrigiano, 2008, 2012; Davis, 2013). At presynaptic sites, homeostasis encompasses the fast, long-lasting and accurate modulation of synaptic vesicle fusion (Davis, 2013). Alteration at protein level as response to homeostatic scaling in hippocampal neurons was recently analyzed by Schanzenbächer et al. (2016). They uncovered the necessity of new protein synthesis upon upor down scaling induced by pharmacological treatment. More than 300 proteins (e.g., neurotransmitter receptors, scaffolding and signaling proteins) were affected by this stimulation. Strikingly, genes affected by the stimulation, encode for proteins critically involved in neurological diseases like AD, Parkinson's disease (PD) or schizophrenia. Proteins identified and regulated by homeostatic scaling in this approach provide a starting point to examine how their dysregulation might contributes to a variety of neuronal disorders (Schanzenbächer et al., 2016).

\section{APP AND THE HIPPOCAMPUS}

APP is functionally integrated into the hippocampal PAZ proteome and fits into the evolutionary conserved active zone protein complex, comprising prominent constituents like ELKS, CASK bassoon, RIM and Munc18 (Südhof, 2012; Laßek et al., 2016). Embedding APP into the entire PAZ proteome unraveled APP as a context-sensitive regulator with impact on synaptic vesicle cycle, cytoskeletal organization and $\mathrm{Ca}^{2+}$-homeostasis. Deletion of APP significantly affects those proteins serving as mediator (e.g., $\alpha$-synuclein) within the PAZ but not their central players (e.g., SNARE-machinery). It was obvious, that loss of APP accounts for individual rearrangements of the entire network structure with no current effect on presynaptic functionality (Laßek et al., 2016). Interestingly, these massive alterations in protein abundance within the PAZ proteome did not account for impairments in learning and memory pointing to a yet unknown compensatory mechanism in young APP-KO mice (Ring et al., 2007). The most important guarantors for sufficient energy metabolism, calcium- and redox homeostasis are mitochondria (Yin et al., 2014; Grimm et al., 2016). They support the intracellular energy demand by producing ATP, affect redox-sensitive kinases via second messengers $\mathrm{H}_{2} \mathrm{O}_{2}$ and $\mathrm{NO}$ and regulate the $\mathrm{NAD}+/ \mathrm{NADH}$ homeostasis, involved in maintenance of mitochondrial energy statues (Yin et al., 2014). A recent proteome study on young and old APP-KO mice revealed drastic changes in mitochondrial protein abundance at hippocampal neurotransmitter release sites. These results indicated that old APP-KO mice display a dysregulation in their bioenergetics metabolism accompanied by hyperphosphorylation of CaMKII (Laßek et al., 2017). It is tempting to speculate that during the induction of LTP CaMKII becomes over-activated, which has a negative impact on synaptic plasticity, and prevents proper learning and memory consolidation. Recently, over-activation of CaMKII was described in hippocampal neurons following synaptic stimulation and increased intracellular $\mathrm{Ca}^{2+}$-levels. As a kind of protective mechanism, CaMKII is able to form clusters (spherical clusters, identical in size and shape) preventing excessive protein phosphorylation, independent of the autocatalytic center, due to an imbalance in $\mathrm{Ca}^{2+}$-homeostasis (Dosemeci et al., 2007). Therefore, cognitive impairments in old APP-KO mice might be associated with an imbalance in mitochondrial function and phosphorylation-activity of the serine/threoninespecific kinases CaMKII as observed during the progression of AD (Grimm et al., 2016). Thermodynamic imbalance and compensatory mechanisms acting in impaired neurons, will further induce a competition for energy substrates and finally shift formerly healthy neurons into affected ones (Demetrius et al., 2015).

The expression pattern of APP in the hippocampus and especially at PAZs has been further analyzed in detail by Rodrigues et al. (2014), demonstrating that APP is most abundant on glutamatergic neurotransmitter release sites as compared to GABAergic ones. Their findings further revealed that less than half of hippocampal synapses were immunopositive for APP (Rodrigues et al., 2014). Strikingly, deletion of APP accounts for an increase in activity of GABAergic synapses. This dysregulation in balance between inhibitory and excitatory neurons was induced by a diminished endocytosis of VDCC in GABAergic hippocampal neurons (Yang et al., 2009). In this context it is worth mentioning the idea of graded molecular profiles of hippocampal neurons (here CA1 neurons) stated by Cembrowski et al. (2016). The hippocampal formation has been attributed with regional-specific functions. Whereas the dorsal hippocampus is known to be associated with cognitive functions (like memory and spatial navigation) the ventral region is basically associated with behavior (like stress and emotion; Fanselow and Dong, 2010; Strange et al., 2014). Illustrating the gene expression profile along the dorsal-ventralaxis of CA1 neurons revealed unique profiles of decay. These findings make it rather interesting to figure out how those CA1 neurons perform their region-specific functions. In line with these findings similar considerations were made for the diversity of presynaptic performance. Atwood and Karunanithi (2002) described various models of functional differentiation of presynaptic neurons. (1) Different amounts of strengthregulating presynaptic proteins or a variable combination of more than one presynaptic protein can be induced or attracted by a postsynaptic neuron. (2) Neuronal activity or specific input to presynaptic neurons can induce a differential occurrence of presynaptic proteins in different neurons (Atwood and Karunanithi, 2002). However, if the molecular profile and 
physiology of those neurons is different, what about the profile of individually expressed proteins? The abundance of several PAZ proteins differs considerably between brain regions, presumably reflecting region-specific functional adaptions. This can be of vital importance to understand the impact of therapeutic drugs (e.g., prevalence or therapy of $\mathrm{AD}$ ) on their targets and to elucidate their subsequent effects on the PAZ proteome. The identification of individual PAZ protein components is a prerequisite for further functional investigations and also provides a solid basis for evaluating their interaction. Therefore, differences in the PAZ proteome reflect specific adaptions to regional neuronal circuitries and the functional and structural dynamics of their corresponding release sites (Weingarten et al., 2015). Salient findings by Schwenk et al. (2014) are in accordance with this dynamic and functional diversity of proteomes. They demonstrated that a large multiprotein complex provides an individual assembly of its core-subunits and a regional specific architecture over space and time (Schwenk et al., 2014). This perspective is indispensable for those proteins sharing a differential expression pattern (like APP) - not only in specific brain regions but also in neurons and individual synapses, respectively. A current study by Counts et al. (2014) went a step further, performing molecular profiling of CA1 neurons derived from patients with mild cognitive impairment $(\mathrm{MCI})$ and $\mathrm{AD}-\mathrm{MCI}$, is a prodromal stage of $\mathrm{AD}$. It is widely accepted that early pathological events triggering the outcome of $\mathrm{AD}$ are associated with $\mathrm{CA} 1$ neurons. Compared to control groups (NCI, no cognitive impairments), expression of genes involved in proper synaptic function in CA1 neurons, is severely dysregulation in $\mathrm{MCI}$, whereas no further changes were observed in $\mathrm{AD}$. Interestingly, APP,

\section{REFERENCES}

Atwood, H. L., and Karunanithi, S. (2002). Diversification of synaptic strength: presynaptic elements. Nat. Rev. Neurosci. 3, 497-516. doi: 10.1038/nrn876

Boveris, A., and Navarro, A. (2008). Brain mitochondrial dysfunction in aging. IUBMB Life 60, 308-314. doi: 10.1002/iub.46

Burré, J., and Volknandt, W. (2007). The synaptic vesicle proteome. J. Neurochem. 101, 1448-1462. doi: 10.1111/j.1471-4159.2007.04453.x

Cannon, W. B. (1932). The Wisdom of the Body. New York, NY: W W Norton and Co.

Cembrowski, M. S., Bachman, J. L., Wang, L., Sugino, K., Shields, B. C., and Spruston, N. (2016). Spatial gene-expression gradients underlie prominent heterogeneity of CA1 pyramidal neurons. Neuron 89 , 351-368. doi: 10.1016/j. neuron.2015.12.013

Coulson, E. J., Paliga, K., Beyreuther, K., and Masters, C. L. (2000). What the evolution of the amyloid protein precursor supergene family tells us about its function. Neurochem. Int. 36, 175-184. doi: 10.1016/s0197-0186(99)00125-4

Counts, S. E., Alldred, M. J., Che, S., Ginsberg, S. D., and Mufson, E. J. (2014). Synaptic gene dysregulation within hippocampal CA1 pyramidal neurons in mild cognitive impairment. Neuropharmacology 79, 172-179. doi: 10.1016/j. neuropharm.2013.10.018

Davis, G. W. (2013). Homeostatic signaling and the stabilization of neural function. Neuron 80, 718-728. doi: 10.1016/j.neuron.2013.09.044

Demetrius, L. A., Magistretti, P. J., and Pellerin, L. (2015). Alzheimer's disease: the amyloid hypothesis and the Inverse Warburg effect. Front. Physiol. 5:522. doi: 10.3389/fphys.2014.00522

Dosemeci, A., Makusky, A. J., Jankowska-Stephens, E., Yang, X., Slotta, D. J., and Markey, S. P. (2007). Composition of the synaptic PSD-95 complex. Mol. Cell. Proteomics 6, 1749-1760. doi: 10.1074/mcp.m700040-mcp200
APLP1 and APLP2 transcripts were not altered at any stage in CA1 neurons. Molecular profiling of CA1 neurons revealed that early changes in synaptic elements provide susceptibility to cognitive decline in aged patients. These findings point to an early onset of synaptic failure that becomes manifested in the dysregulation of the hippocampal neuronal circuit (Counts et al., 2014).

\section{CONCLUDING REMARKS}

Our proteomic profiling of PAZs derived from total mouse brain revealed a summary of all alterations due to loss of APP. Going a step further, looking only at the hippocampus, the picture of presynaptic changes was impressively refined. Therefore, the next step should include proteomic studies addressing the molecular profiling of individual neurons within the hippocampus. Combining our approaches with new technologies will provide novel insights into the biological function of APP within the CNS. Moreover, interdisciplinary approaches and sustained exchanges of information can facilitate new perspectives within the challenging APP research field.

\section{AUTHOR CONTRIBUTIONS}

The authors MWei, JW, MWeg and WV contributed equally to this review article.

\section{ACKNOWLEDGMENTS}

We are grateful to Herbert Zimmermann for valuable suggestions.

Eggert, S., Paliga, K., Soba, P., Evin, G., Masters, C. L., Weidemann, A., et al (2004). The proteolytic processing of the amyloid precursor protein gene family members APLP-1 and APLP-2 involves $\alpha-, \beta-, \gamma$ - and $\epsilon$-like cleavages. modulation of APLP-1 processing by n-glycosylation. J. Biol. Chem. 279, 18146-18156. doi: 10.1074/jbc.m311601200

Fanselow, M. S., and Dong, H.-W. (2010). Are the dorsal and ventral hippocampus functionally distinct structures? Neuron 65, 7-19. doi: 10.1016/j.neuron.2009. 11.031

Fitzjohn, S. M., Morton, R. A., Kuenzi, F., Davies, C. H., Seabrook, G. R., and Collingridge, G. L. (2000). Similar levels of long-term potentiation in amyloid precursor protein -null and wild-type mice in the CA1 region of picrotoxin treated slices. Neurosci. Lett. 288, 9-12. doi: 10.1016/s0304-3940(00) 01204-0

Glenner, G. G., and Wong, C. W. (1984). Alzheimer's disease and down's syndrome. sharing of a unique cerebrovascular amyloid fibril protein. Biochem. Biophys. Res. Commun. 122, 1131-1135. doi: 10.1016/0006-291x(84) 91209-9

Gray, E. G. (1963). Electron microscopy of presynaptic organelles of the spinal cord. J. Anat. 97, 101-106.

Grimm, A., Friedland, K., and Eckert, A. (2016). Mitochondrial dysfunction: the missing link between aging and sporadic Alzheimer's disease. Biogerontology 17, 281-296. doi: 10.1007/s10522-015-9618-4

Groemer, T. W., Thiel, C. S., Holt, M., Riedel, D., Hua, Y., Hüve, J., et al. (2011). Amyloid precursor protein is trafficked and secreted via synaptic vesicles. PLoS One 6:e18754. doi: 10.1371/journal.pone.0018754

Grundke-Iqbal, I., Iqbal, K., Tung, Y. C., Quinlan, M., Wisniewski, H. M., and Binder, L. I. (1986). Abnormal phosphorylation of the microtubule-associated protein tau (tau) in Alzheimer cytoskeletal pathology. Proc. Natl. Acad. Sci. US A 83, 4913-4917. doi: 10.1073/pnas.83.13.4913 
Heber, S., Herms, J., Gajic, V., Hainfellner, J., Aguzzi, A., Rülicke, T., et al. (2000). Mice with combined gene knock-outs reveal essential and partially redundant functions of amyloid precursor protein family members. J. Neurosci. 20, 7951-7963.

Hick, M., Herrmann, U., Weyer, S. W., Mallm, J.-P., Tschäpe, J.-A., Borgers, M., et al. (2015). Acute function of secreted amyloid precursor protein fragment APPs $\alpha$ in synaptic plasticity. Acta Neuropathol. 129, 21-37. doi: 10.1007/s00401-014-1368-x

Jacobsen, K. T., and Iverfeldt, K. (2009). Amyloid precursor protein and its homologues. a family of proteolysis-dependent receptors. Cell. Mol. Life Sci. 66, 2299-2318. doi: 10.1007/s00018-009-0020-8

Kaden, D., Munter, L. M., Reif, B., and Multhaup, G. (2012). The amyloid precursor protein and its homologues. structural and functional aspects of native and pathogenic oligomerization. Eur. J. Cell Biol. 91, 234-239. doi: 10.1016/j.ejcb.2011.01.017

Kang, J., Lemaire, H. G., Unterbeck, A., Salbaum, J. M., Masters, C. L., Grzeschik, K. H., et al. (1987). The precursor of Alzheimer's disease amyloid A4 protein resembles a cell-surface receptor. Nature 325, 733-736. doi: $10.1038 / 325733 \mathrm{a} 0$

Korte, M., Herrmann, U., Zhang, X., and Draguhn, A. (2012). The role of APP and APLP for synaptic transmission, plasticity, and network function. lessons from genetic mouse models. Exp. Brain Res. 217, 435-440. doi: 10.1007/s00221-0112894-6

Laßek, M., Weingarten, J., Acker-Palmer, A., Bajjalieh, S. M., Muller, U., and Volknandt, W. (2014). Amyloid precursor protein knockout diminishes synaptic vesicle proteins at the presynaptic active zone in mouse brain. Curr. Alzheimer Res. 11, 971-980. doi: 10.2174/1567205011666141107152458

Laßek, M., Weingarten, J., Einsfelder, U., Brendel, P., Müller, U., and Volknandt, W. (2013). Amyloid precursor proteins are constituents of the presynaptic active zone. J. Neurochem. 127, 48-56. doi: 10.1111/jnc.12358

Laßek, M., Weingarten, J., and Volknandt, W. (2015). The synaptic proteome. Cell Tissue Res. 359, 255-265. doi: 10.1007/s00441-014-1943-4

Laßek, M., Weingarten, J., Wegner, M., Mueller, B. F., Rohmer, M., Baeumlisberger, D., et al. (2016). APP is a context-sensitive regulator of the hippocampal presynaptic active zone. PLoS Comput. Biol. 12:e1004832. doi: 10.1371/journal.pcbi.1004832

Laßek, M., Weingarten, J., Wegner, M., Neupärtl, M., Array, T. N., Harde, E., et al. (2017). APP deletion accounts for age-dependent changes in the bioenergetic metabolism and in hyperphosphorylated CaMKII at stimulated hippocampal presynaptic active zones. Front. Synaptic Neurosci. 9:1. doi: 10.3389/fnsyn.2017. 00001

Lazarov, O., and Demars, M. P. (2012). All in the family: how the APPs regulate neurogenesis. Front. Neurosci. 6:81. doi: 10.3389/fnins.2012.00081

Lu, J. X., Qiang, W., Yau, W. M., Schwieters, C. D., Meredith, S. C., and Tycko, R. (2013). Molecular structure of $\beta$-amyloid fibrils in Alzheimer's disease brain tissue. Cell 154, 1257-1268. doi: 10.1016/j.cell.2013.08.035

Lyckman, A. W., Confaloni, A. M., Thinakaran, G., Sisodia, S. S., and Moya, K. L. (1998). Post-translational processing and turnover kinetics of presynaptically targeted amyloid precursor superfamily proteins in the central nervous system. J. Biol. Chem. 273, 11100-11106. doi: 10.1074/jbc.273.18.11100

Mann, E. O., and Paulsen, O. (2007). Role of GABAergic inhibition in hippocampal network oscillations. Trends Neurosci. 30, 343-349. doi: 10.1016/j. tins.2007.05.003

Marquez-Sterling, N. R., Lo, A. C., Sisodia, S. S., and Koo, E. H. (1997). Trafficking of cell-surface $\beta$-amyloid precursor protein. evidence that a sorting intermediate participates in synaptic vesicle recycling. J. Neurosci. 17, 140-151.

Mattson, M. P., Gleichmann, M., and Cheng, A. (2008). Mitochondria in neuroplasticity and neurological disorders. Neuron 60, 748-766. doi: 10.1016/j. neuron.2008.10.010

Phinney, A. L., Calhoun, M. E., Wolfer, D. P., Lipp, H. P., Zheng, H., and Jucker, M. (1999). No hippocampal neuron or synaptic bouton loss in learning-impaired aged $\beta$-amyloid precursor protein-null mice. Neuroscience 90, 1207-1216. doi: 10.1016/s0306-4522(98)00645-9

Ring, S., Weyer, S. W., Kilian, S. B., Waldron, E., Pietrzik, C. U., Filippov, M. A., et al. (2007). The secreted $\beta$-amyloid precursor protein ectodomain APPs $\alpha$ is sufficient to rescue the anatomical, behavioral, and electrophysiological abnormalities of APP-deficient mice. J. Neurosci. 27, 7817-7826. doi: 10.1523/JNEUROSCI.1026-07.2007
Rodrigues, D. I., Gutierres, J., Pliássova, A., Oliveira, C. R., Cunha, R. A., and Agostinho, P. (2014). Synaptic and sub-synaptic localization of amyloid- $\beta$ protein precursor in the rat hippocampus. J. Alzheimers Dis. 40, 981-992. doi: 10.3233/JAD-132030

Schanzenbächer, C. T., Sambandan, S., Langer, J. D., and Schuman, E. M. (2016). Nascent proteome remodeling following homeostatic scaling at hippocampal synapses. Neuron 92, 358-371. doi: 10.1016/j.neuron.2016. 09.058

Schwenk, J., Baehrens, D., Haupt, A., Bildl, W., Boudkkazi, S., Roeper, J., et al. (2014). Regional diversity and developmental dynamics of the AMPA-receptor proteome in the mammalian brain. Neuron 84, 41-54. doi: 10.1016/j.neuron. 2014.08.044

Strange, B. A., Witter, M. P., Lein, E. S., and Moser, E. I. (2014). Functional organization of the hippocampal longitudinal axis. Nat. Rev. Neurosci. 15, 655-669. doi: 10.1038/nrn3785

Südhof, T. C. (2012). The presynaptic active zone. Neuron 75, 11-25. doi: 10.1016/j.neuron.2012.06.012

Südhof, T. C., and Rizo, J. (2011). Synaptic vesicle exocytosis. Cold Spring Harb. Perspect. Biol. 3:a005637. doi: 10.1101/cshperspect.a005637

Supnet, C., and Bezprozvanny, I. (2010). The dysregulation of intracellular calcium in Alzheimer disease. Cell Calcium 47, 183-189. doi: 10.1016/j.ceca.2009.12.014

Turrigiano, G. (2008). The self-tuning neuron: synaptic scaling of excitatory synapses. Cell 135, 422-435. doi: 10.1016/j.cell.2008.10.008

Turrigiano, G. (2012). Homeostatic synaptic plasticity: local and global mechanisms for stabilizing neuronal function. Cold Spring Harb. Perspect. Biol. 4:a005736. doi: 10.1101/cshperspect.a005736

Tyan, S. H., Shih, A. Y., Walsh, J. J., Maruyama, H., Sarsoza, F., Ku, L., et al. (2012). Amyloid precursor protein (APP) regulates synaptic structure and function. Mol. Cell. Neurosci. 51, 43-52. doi: 10.1016/j.mcn.2012.07.009

Weingarten, J., Laßek, M., Mueller, B., Rohmer, M., Baeumlisberger, D., Beckert, B., et al. (2015). Regional specializations of the paz proteomes derived from mouse hippocampus, olfactory bulb and cerebellum. Proteomes 3, 74-88. doi: 10.3390/proteomes3020074

Weyer, S. W., Klevanski, M., Delekate, A., Voikar, V., Aydin, D., Hick, M., et al. (2011). APP and APLP2 are essential at PNS and CNS synapses for transmission, spatial learning and LTP. EMBO J. 30, 2266-2280. doi: 10.1038/emboj.2011.119

Weyer, S. W., Zagrebelsky, M., Herrmann, U., Hick, M., Ganss, L., Gobbert, J., et al. (2014). Comparative analysis of single and combined APP/APLP knockouts reveals reduced spine density in APP-KO mice that is prevented by APPs $\alpha$ expression. Acta Neuropathol. Commun. 2:36. doi: 10.1186/20515960-2-36

Yang, L., Wang, Z., Wang, B., Justice, N. J., and Zheng, H. (2009). Amyloid precursor protein regulates Cav1.2 L-type calcium channel levels and function to influence GABAergic short-term plasticity. J. Neurosci. 29, 15660-15668. doi: 10.1523/JNEUROSCI.4104-09.2009

Yin, F., Boveris, A., and Cadenas, E. (2014). Mitochondrial energy metabolism and redox signaling in brain aging and neurodegeneration. Antioxid. Redox Signal. 20, 353-371. doi: 10.1089/ars.2012.4774

Yu, L. M. Y., and Goda, Y. (2009). Dendritic signalling and homeostatic adaptation. Curr. Opin. Neurobiol. 19, 327-335. doi: 10.1016/j.conb.2009. 07.002

Conflict of Interest Statement: The authors declare that the research was conducted in the absence of any commercial or financial relationships that could be construed as a potential conflict of interest.

The handling Editor declared a shared affiliation, though no other collaboration, with the authors JW, MWei, MWeg and WV, and the handling Editor states that the process met the standards of a fair and objective review.

Copyright (c) 2017 Weingarten, Weingarten, Wegner and Volknandt. This is an open-access article distributed under the terms of the Creative Commons Attribution License (CC BY). The use, distribution and reproduction in other forums is permitted, provided the original author(s) or licensor are credited and that the original publication in this journal is cited, in accordance with accepted academic practice. No use, distribution or reproduction is permitted which does not comply with these terms. 